\title{
ЗАПАЗДЫВАЮЩЕЕ И ОПЕРЕЖАЮЩЕЕ ЭХО
}

1. HIZNJAKOV. EEL- JA JARELKAJA

1. HIZHNYAKOV. RETARDED AND ADVANCED ECHO

1. В системах с неоднородно уширенными спектрами поглощения наблюдается эхо - импульс отклика на возбуждение несколькими импульсами $\left[{ }^{1,2}\right]$. Простейшее явление такого типа - стимулированное эхо (СЭ) - четвертый импульс, испускаемый системой после возбуждения гремя короткими импульсами []. Физическая причина СЭ связана с запомннанием системой спсктра действующей раднации (обычное время запоминания $t_{0}$ определяется временем жизни возбужденного состояния, а в системах с выжиганием провала - временем жизни провала $\left.\left[{ }^{4}\right]\right)$. Спектр $I(\omega)$ двух коротких импульсов, разделенных временным интервалом $\tau$, промодулирован по частоте множителем $(1+\cos \omega \tau)$

$$
I(\omega)=\left|\int_{-\infty}^{\infty} d t E_{l}(t) e^{-i \omega t}\left(1+e^{i \omega \tau}\right)\right|^{2}=2\left|E_{l}(\omega)\right|^{2}(1+\cos \omega \tau)
$$

$\left(E_{l}(t)\right.$ - напряженность поля одного импульса, $E_{l}(\omega)$ - ее Фурьеобраз). Поэтому аналогичная модуляция возникает на время $t_{0}$ и в спектре поглощения системы. Эта модуляция проявляется при пропускании третьего импульса в виде эхо-сигнала - четвертого импульса.

Характерным свойстеом СЭ является равенство времен задержки между первой и второй парами импульсов: $\tau_{43}=\tau_{21}=\tau$. Отсюда, в частности, следует совпадение форм (с точностью до инверсии) кодирующего импульса (более слабого из первых двух) и эхо-сигнала. Указанное свойство обусловлено совпадением спектров (точнее, гамильтонианов) системы в промежутках $\tau_{21}$ и $\tau_{3}$ между импульсами (в эти промежутки времени отличаются лишь населенности уровней, но не их энергии). Если с помощью какого-либо внешнего воздействия на систему это условие нарушить, то нарушится и отмеченное равенство. При этом форма импульса эхо-сигнала будет, вообще говоря, отличаться от формы кодирующего импульса.

Если отмеченное выше воздействие подобрать так, что энергетические спектры системы в интервалах $\tau_{21}$ и $\tau_{43}$ будут связаны линейным преобразованием, то линейно будут связаны и сами интервалы $\tau_{21}$ и $\tau_{43}$. При этом эхо будет восстанавливать (с точностью до инверсии) форму кодирующего импульса в измененном временном масштабе. В частности, если энсргетический спектр сжат в интервале $\tau_{21}$, то импульс эхосигнала растянут во времени, а если указанный спектр сжат в интервале $\tau_{43}$, то и импульс его сжат во времени. Ниже мы покажем, что обе эти ситуации, соответствующие запаздывающему и опережающему СЭ, могут быть реализованы при использовании в качестве внешнего воздействия интенсивного (лазерного) резонансного излучения.

2. Предположим, что лазерное поле находится в резонансе с переходом 
между невырожденным и двукратно квазивырожденным состояниями атома (примесного центра кристалла). Примем для определенности, что квазивырожден верхний уровень. Считаем, что переходы $|1\rangle_{a} \rightarrow|2\rangle_{a}$ и $|1\rangle_{a} \rightarrow|3\rangle_{a}$ разрешены в разных поляризациях, а соответствующие полосы поглощения неоднородно уширены вследствие разброса значений частоты $\omega \equiv\left(E_{2}+E_{3}\right) / 2-E_{1}$ по ансамблю центров $\left(\hbar=1,|n\rangle_{a}-\right.$ состояния центра, $E_{n}$ - их энергии); в случае свободных атомов роль неоднородного уширения играет допплеровское уширение. Расщепление возбужденных уровней $2 \delta=E_{3}-E_{2}$ считается одинаковым для всех атомов.

В интенсивном поле частоты $\omega_{L} \approx \omega$ волновые функции и энергии атомных состояний изменяются. Указанные изменения можно найти методом одетых состояний $\left[{ }^{5}\right]$ из решения задачи на собственные состояния $\left|\psi_{i}\right\rangle=\sum_{n} c_{i n}|n\rangle$ и собственные значения $\varepsilon_{i}$ матрицы энергии

$$
\hat{\varepsilon}=\left|\begin{array}{ccc}
\omega_{L}-\omega & \Gamma_{1} & \Gamma_{2} \\
\Gamma_{1} & -\delta & 0 \\
\Gamma_{2} & 0 & \delta
\end{array}\right|,
$$

представляющей собой блок матрицы-гамильтониана системы атома и лазерного поля, записанной в базисе состояний $|1\rangle=|1\rangle_{x}|N\rangle,|2\rangle=$ $=|2\rangle_{a}|N-1\rangle,|3\rangle=|3\rangle_{a}|N-1\rangle$. Здесь $|N\rangle-N$-фотонное состояние лазерной моды, $\Gamma_{1} \sim d_{12} \sqrt{N}$ и $\Gamma_{2} \sim d_{13} \sqrt{N} \quad$ - энергии взаимодействия переходов $|1\rangle_{a} \rightarrow|2\rangle_{a}$ и $|1\rangle_{a} \rightarrow|3\rangle_{a}$ с лазерным полем, $d_{12}$ и $d_{13}-$ матричные элементы соответствующих переходов; считается, что $N \gg 1$; за начало отсчета принята энергия $E_{0}=E_{1}+(N-1) \omega_{L}+\omega$. Состояния, диагонализующие матрицу (2), называются одетыми [5].

3. Рассмотрим, как изменяется СЭ под действием интенсивного резонансного лазерного излучения. Для этого следует определить волновые функции и энергии одетых состояний. Последние являются корнями уравнения

$$
\varepsilon^{3}-\omega_{0} \varepsilon^{2}-\Omega_{0}^{2} \varepsilon+\delta\left(\Gamma_{1}^{2}-\Gamma_{2}^{2}+\omega_{0} \delta\right)=0,
$$

где $\omega_{0}=\omega_{L}-\omega$,

$$
\Omega_{0}=\left(\Gamma_{1}^{2}+\Gamma_{2}^{2}+\delta^{2}\right)^{1 / 2}
$$

- частота Раби. В пределе сильного поля $\left(\left|\Gamma_{1}\right| \gg|\delta|\right.$ либо $\left.\left|\Gamma_{2}\right| \gg|\delta|\right)$ для малых $\left|\omega_{L}-\omega\right| \ll \Omega_{0} \approx\left(\Gamma_{1}^{2}+\Gamma_{2}^{2}\right)^{1 / 2}$ эти корни следующне:

$$
\begin{aligned}
& \varepsilon_{1} \approx \varepsilon_{0}+\left(\omega_{L}-\omega\right) \delta^{2} / \Omega_{0}^{2}, \\
& \varepsilon_{2,3} \approx \varepsilon_{0} \mp \Omega_{0}+\left(\omega_{L}-\omega\right) / 2,
\end{aligned}
$$

где $\varepsilon_{0}=\delta\left(\Gamma_{1}^{2}-\Gamma_{2}^{2}\right) / \Omega_{0}^{2}$. Соответствующие волновые функции равны:

$$
\begin{aligned}
& \left|\psi_{1}\right\rangle=\alpha|1\rangle_{a}|N\rangle+\cos \varphi|2\rangle_{a}|N-1\rangle-\sin \varphi|3\rangle_{a}|N-1\rangle, \\
& \left|\psi_{2}\right\rangle=\frac{1}{\sqrt{2}}\left(|1\rangle_{a}|N\rangle-\sin \varphi|2\rangle_{a}|N-1\rangle-\cos \varphi|3\rangle_{a}|N-1\rangle\right), \\
& \left|\psi_{3}\right\rangle=\frac{1}{\sqrt{2}}\left(|1\rangle_{a}|N\rangle+\sin \varphi|2\rangle_{a}|N-1\rangle+\cos \varphi|3\rangle_{a}|N-1\rangle\right),
\end{aligned}
$$

где $\varphi=\arctan \left(\Gamma_{1} / \Gamma_{2}\right), \alpha=\sqrt{2} \delta \Gamma_{1} \Gamma_{2} \Omega_{0}^{-3}$. 
При адиабатически медленном (по сравнению с $\Gamma^{-1}$ ) включении лазерного поля атомы, находящиеся в основном состоянии, попадут в одетое состояние $\left|\psi_{1}\right\rangle$, а атомы, находящиеся в возбужденном состоянии $|2\rangle_{a}$, попадут в одетое состояние $\left|\psi_{2}\right\rangle$. При этом пробное излучение поляризации, отличной от (перпендикулярной) поляризации лазерного поля, будет усиливаться (если заселено состояние $\left.\left|\psi_{1}\right\rangle\right)$, либо поглощаться (если заселено состояние $\left.\left|\psi_{2}\right\rangle\right)$ на частоте $\Omega_{1} \approx\left(\omega+\omega_{L}\right) / 2-\Omega_{0}$. Кроме того, возникает поглощение (усиление) на частоте $\Omega_{2} \approx$ $\approx \omega_{L}+2\left[\Gamma_{1}^{2}+\Gamma_{2}^{2}+\left(\omega-\omega_{L}\right)^{2}\right]^{1 / 2}$ и усиление (поглощение) на частоте $\Omega_{3} \approx \omega_{L}-2\left[\Gamma_{1}^{2}+\Gamma_{2}^{2}+\left(\omega-\omega_{L}\right)^{2}\right]^{1 / 2}$. Соответствующие полосы поглощения-усиления сжимаются по сравнению с полосой поглощения в отсутствии лазерного поля; коэффициент сжатия равен двум для первой полосы, а для второй и третьей полос он равен $\beta=\left[\left(\omega_{1}-\omega_{L}\right)^{2} /\left(\Gamma_{1}^{2}+\Gamma_{2}^{2}+\right.\right.$. $\left.\left.+\left(\omega_{1}-\omega_{L}\right)^{2}\right)\right]^{1 / 2}$, где $\omega_{1}-$ частота актуального для СЭ участка спектра поглощения в отсутствии лазерного поля. Поэтому время возникновения сигнала СЭ изменяется в два либо в $\beta$ раз в зависимости от несущей частоты импульса (ов) возбуждения СЭ, действующего (их) одновременно с лазерным импульсом.

Отметим, что двухуровневые системы также могут быть использованы для получения запаздывающего и опережающего СЭ. В таких системах степень опережения-запаздывания СЭ равна $\beta=\left[\left(\omega_{1}-\omega_{L}\right)^{2} /\left(\Gamma_{1}^{2}+\right.\right.$ $\left.+\left(\omega_{1}-\omega_{L}\right)^{2}\right]^{1 / 2} \quad\left(\right.$ здесь $\left.\delta=\Gamma_{2}=0\right)$.

4. Рассмотренные задержка и опережение СЭ с помощью интенсивного резонансного лазерного излучения могут быть осуществлены не только в примесных кристаллах, но и в газах на допплеровски уширенных линиях поглощения. Интересно отметить, что в газовых струях запаздывание и опережение сигналов СЭ могут быть получены и без интенсивного лазерного излучения. Здесь шаг записанной первыми двумя импульсами и считываемой третьим импульсом спектральной решетки зависит как от направлений возбуждающих (первых двух), так и направления считываемого (третьего) импульсов. Поэтому коэффициент запаздывания (опережения) СЭ оказывается равным отношению $\beta=$ $=\left|\cos \varphi_{1} / \cos \varphi_{3}\right|$, где $\varphi_{1}$ - угол между направлением первых двух импульсов и струи, а $\varphi_{3}$ - угол между направлением третьего импульса и струи. Если $\left|\varphi_{1}\right|<\left|\varphi_{3}\right|<\pi / 2$, то $\beta>1$ и СЭ является запаздывающим, а если $\left|\varphi_{3}\right|<\left|\varphi_{1}\right|<\pi / 2$, то $\beta<1$ и СЭ опережающее.

5. Примером конкретной системы, на которой можно наблюдать рассмотренные здесь эффекты запаздывающего и опережающего СЭ, может служить пар атомарного натрия, имеющего подходящие оптические переходы - сверхтонкие компоненты желтой $(D)$ линии. Условие $\Gamma \geqslant \delta \sim 10^{-3} \mathrm{~cm}^{-1}$ выполняется при использовании лазерного импульса пиковой мощностью $\sim 10^{3} \mathrm{BT} / \mathrm{cm}^{2}$. Запаздывающее и опережающее СЭ можно возбуждать тремя слабыми импульсами длительностью $<10^{-9}$ с, с интервалами между ними $\tau_{32}>\tau_{21} \sim 10^{-9}$ с и интенсивным импульсом длительностью $\sim 10^{-9}$ с. Эффект можно наблюдать и на компонентах разрешенных переходов других атомов. В кристаллах можно использовать чистоэлектронные линии примесиых центров при низкой температуре. В этом случае параметры лазерного импульса должны быть примерно такими же, как.и в случае натрия.

В заключение отметим, что рассмотренные здесь эффекты запаздывающего и опережающего эхо-сигналов могут наблюдаться и в системах с выжиганием провала. Кроме того, аналогичные эффекты могут также возникать и в случае обычного (т. н. первичного) эхо-сигнала, возбуждаемого двумя импульсами. Эти эффекты могут также быть использо- 
ваны для ускоренного либо замедленного воспроизведения событий по методу пространственно-временной голографии $\left[{ }^{4,6}\right]$, т. е. для реализации временного микроскопа и временного телескопа.

\section{ЛИ Т Е Р А Т У Р А}

1. Kurnit, N. A., Abella, I. D., Hartmann, S. R. Phys. Rev. Lett., 13, 567-568 (1964)

2. Аллен Л., Эберли Дж. Оптический резонанс и двухуровнезые атомы. М., «Мир», 1978.

3. Mosberg, T. W., Kachru, R., Hartman, S. R., Flusberg, A. Phys. Rev. A., 20, 1976-1996 (1979).

4. Саари П., Ребане А. Изв. АН ЭССР. Физ. Матем., 33, № 3, 322-332 (1984).

5. Knight, P. L., Milonni, P. W. Phys. Rev., 66, 21-107 (1980).

6. Mosberg, T. W. Opt. Lett., 7, № 2, 77-79 (1982).
Институт физики
Академии наук Эстонской ССР
Поступила в редакцию $29 / X-1985$ 